OAI-PMH: http://www.indteca.com/ojs/index.php/Revista Scientific/oai

Ensayo Original / Original Essay

\title{
Nociones Críticas en la Responsabilidad Social de la Extensión Universitaria con la Vinculación Comunidad
}

Autora: Mariella Fidelina Rojas Zerpa

Universidad Pedagógica Experimental Libertador, UPEL

mariella1117@gmail.com

Lara, Venezuela

\section{Resumen}

La responsabilidad social, sistematiza perspectivas que la sociedad posee de las organizaciones, en este caso, las universitarias, en un punto dado en el tiempo, porque superan modelos de efecto mutuo conjuntamente con la función de la extensión desafiando ante un meditar completo de la institución, basado en una fundación sabia interventora desde los contextos circundantes. Es por eso que, el presente ensayo asumió como propósito analizar las nociones críticas en la responsabilidad social de la extensión universitaria con la vinculación comunidad. La metodología utilizada se basó en la síntesis de lo analizado con argumentación propia de la autora además de reflexiones que pudieran puedan ser un aporte para el proceso de ofrecer alternativas ayudando así a solucionar problemáticas del entorno social. De manera concluyente el éxito universitario en este momento consiste el formar el profesorado tomando en consideración la noción de responsabilidad e integralidad para propiciar trabajos fusionando las diversas áreas académicas.

Palabras clave: responsabilidad social; extensión universitaria; comunidad. 


\title{
Critical Notions in the Social Responsibility of the University Extension with the Community Linkage
}

\begin{abstract}
Social responsibility, systematizes perspectives that society has of organizations, in this case, the universities, at a given point in time, because they overcome models of mutual effect together with the function of extension challenging before a full meditation of the institution, based on a wise interventionist foundation from the surrounding contexts. That is why, the present essay assumed as a purpose to analyze the critical notions in the social responsibility of the university extension with the community connection. The methodology used was based on the synthesis of what was analyzed with the author's own arguments, as well as reflections that could be a contribution to the process of offering alternatives, thus helping to solve problems in the social environment. In a conclusive way, the university success at this moment consists in the formation of the teaching staff taking into consideration the notion of responsibility and integrality to promote works merging the diverse academic areas.
\end{abstract}

Keywords: social responsibility; university extension; community.

Date Received: 13-04-2018

Date Acceptance: 03-07-2018 


\section{Introducción}

En nuestros días, se instituye una necesidad básica instruir en la reafirmación de costumbres, porque ellas son la base formativa en la línea de nuestro sistema de valores y componen un motor impulsor en la cohesión social y el compromiso individual del hombre. Por esto, la responsabilidad social de la extensión universitaria en su vinculación con la comunidad, se ve plasmada hoy en la política educacional venezolana imponiendo nuevos retos, pertinentes de reflexionar ante las nuevas realidades y desafíos que enfrentan las comunidades.

Hablar de caracterización, de la responsabilidad social que compete a la extensión universitaria implica el acompañamiento de un marco contextual referido a la temática a tratar en el ensayo presentado, el objetivo de demarcar el problema es disminuir el grado de complejidad de éste, para concentrarse en los elementos esenciales. De ahí que, los retos y desafíos a enfrentar las universidades y los individuos en el marco de la actualidad, van dirigidos a plantear una reflexión sobre este tiempo, su esencia, los valores que propugna y el rol de la educación en el contexto inherente a esta época.

Visto desde esta perspectiva, es primordial en el contexto universitario, esbozar un proceso reflexivo en cuanto a la incidencia de la ideología postmoderna en la educación, en cuanto a la temática referida en el estudio, debido a que este pensamiento desordena y perturba lo establecido en los diferentes elementos del sistema social.

De hecho, en los novísimos tiempos, la responsabilidad social a juicio de Barañano (2009), citado en Gaete (2012), señala que:

en la década de los noventa la responsabilidad social adquiere un auge y relevancia mayor, debido a los efectos que van alcanzando los nuevos riesgos de la globalización y la transnacionalización empresarial, reflejados en diferentes escándalos financieros, sociales o medio ambientales... (pág. 20). 
Se puede observar hace tiempo, esta idea es acogida. Sin embargo, las instituciones universitarias recientemente es que la comienzan a aceptar como objetivo de la visión que desean lograr en el ámbito que les corresponda abarcar. Sin embargo, no debe ser avocada en la radicalidad sino más bien debe ser acogido como de manera responsable y no como una simple manera de resolver las problemáticas que le atañen.

Por esto, con el presente ensayo se pretende analizar la responsabilidad social que compete a la extensión universitaria en el proceso de vincular a ésta con la comunidad. De todos modos, se observa que en los recintos universitarios debe haber interacción con los ámbitos sociales adyacentes. Tal como lo establece, Rodríguez (2010): cuando expresa que se "deben establecer relaciones con la sociedad, que les permita consolidar su quehacer, cada vez más trascendente para diferentes personas, grupos e instituciones del Estado, y la Sociedad Civil" (pág. 15). En otras palabras, se escuchan diatribas si la universidad es quien debe adecuarse a la sociedad $o$ contrariamente si la colectividad debe adaptarse a particularidades que sean inherentes a la misión educativa de éstas.

En resumidas cuentas, el descrito escenario sirve para adentrarse según Cruz (2010): "desde diferentes perspectivas que intentan establecer y delimitar de qué es responsable la universidad en el siglo XXI, porque habitualmente dichas responsabilidades son influenciadas por la perspectiva económica asignada al quehacer universitario" (pág. 38). Es decir, la universidad se responsabiliza de la sociedad, además de aspectos de su misión en cuanto a lo cultural y sociable, como bien público dirigidos a la excelencia e investigación.

\section{Desarrollo}

La educación universitaria en este momento, su éxito radica en la praxis formativa del profesor actual, sobre todo aquel que es creador de 
generaciones futuristas el cual tiene como misión desplegar actividades tomando en cuenta la noción de integralidad, responsabilidad donde trabaje con diversas áreas académicas de manera integral. Aunado a esto, como profesional debe conocer cada una de ellas de manera individual alcanzando así certidumbre en el trabajo realizado, por lo cual demanda de una preparación y conocimiento académico, para lograr tal propósito cuando se inserta en el campo laboral educativo.

Desde estas ideas, hablar de universidad conduce al término cosmos, sinónimo de unidad convertido en uno. El significado etimológico de la palabra universidad, apunta a la unión de elementos anteriormente dispersos, constituyen la asociación; cuerpo, gremio, centro. De hecho, las universidades enfrentan hoy en día el reto de ser la institución académica orientada al desarrollo de las tres tareas fundamentales necesarias en todo proceso social como son: la enseñanza y formación de nuevas generaciones; la investigación, creación, mantenimiento y difusión de conocimientos; y servir de modelo a la sociedad en cuanto a organización comunitaria, intelectual y democrática del conocimiento. Esta última tarea, debe estar, cuando se develan vinculaciones de la universidad con la comunidad.

En este momento, la política educacional venezolana universitaria impone nuevos retos, pertinentes de reflexionar ante las nuevas realidades y provocaciones que se enfrenta a nivel mundial. En el orden económico, político y social, han trascendido socialmente en la familia venezolana por la visión de determinados fenómenos que transgreden también, en comportamientos impropios que han provocado inadaptación social. Lo que demanda un replanteo de la política educativa que satisfaga las nuevas exigencias y sin perder la esencia de la política expuesta, concertar la realidad presente, con una proyección de futuro, resguardando los valores de justicia social, responsabilidad, soberanía nacional e independencia, y así lograr el mayor desarrollo de la espiritualidad del ser humano. 
Por esto, la Organización de las Naciones Unidas para la Educación, la Ciencia y la Cultura, UNESCO (2008), afirma al respecto: "La enseñanza tiene que preparar a titulados con nuevas destrezas, una amplia base de conocimientos y diversas competencias para moverse en un mundo más complejo e interdependiente...y compartir por encima de las fronteras y en las distintas culturas" (pág. 114). Es decir, considerando que la educación universitaria, en este momento debe enfrentar nuevo rol de estudiante que requiere nuevas responsabilidades para su desempeño laboral y en su vida.

De hecho, en el proceso político y educativo actual que lidera el Sistema Educativo Bolivariano (SEB), es vital el proceso de enseñanza, formación y desarrollo de los valores morales, el cual no está exento de dificultades que debemos atender desde la investigación científica. Por esto, se hace necesario, estar preparados ante los desafíos de las más jóvenes generaciones, y que afectan el proceso de formación de valores, sus relaciones interpersonales y sus modos de actuación en los diferentes contextos donde interactúan. Por esta razón se le da suma importancia a la profundización en el estudio de los problemas sociales. En este sentido, Alonso (1997), citado en Castro y Guzmán (2005), afirma que:

El pensamiento axiológico va más allá del simple reflejo de las relaciones sociales, se construyen como complejidad en la que se entrelazan fenómenos de tipo colectivo e individual. Dialéctica que obliga a no fijarlo y reducirlo exclusivamente a su condicionamiento social, sino ir más allá, y comprenderlo como resultado de las interrelaciones del individuo y la sociedad. Por ello, en el pensamiento axiológico deben incluirse, de alguna manera, los sistemas subjetivos de valores, en relación dialéctica con los valores objetivos de la sociedad en su conjunto (pág. 94).

En el caso mostrado, la dimensión axiológica en este momento presenta matices diversos en cuanto a la responsabilidad social como espacio ético que toda organización o institución debería tener para adaptarlo a modo de 
perspectiva en su quehacer cotidiano, aunque en el ámbito universitario recién empieza a darse.

Por esto, dentro de las investigaciones que examinan las desiguales teorías concernidas con el concepto de responsabilidad social, resulta atractivo a nuestro juicio reseñar el trabajo de Secchi (2007): quien realiza "un aporte muy interesante para clarificar y clasificar las diferentes tendencias, de los conceptos y propuestas desarrolladas en relación con la responsabilidad social observadas en las últimas décadas" (pág. 351).

En otras palabras, la visión integral en las recientes reformas y tendencias de las universidades puede llegar a causar impacto en los componentes de éstas si se enfocan desde la progresividad hacia el emprendimiento de una transformación, dirigida a sustentar el bien común desde la perspectiva de la responsabilidad como influencia socializadora.

Como complemento de la temática, la Educación Integral de acuerdo con el documento final de la Conferencia Mundial sobre los Derechos Humanos (ONU, 1933), establece: "es una educación que sea capaz de preparar hombres autónomos desde el punto de vista moral, respetuoso de la libertad, la dignidad del otro; he aquí el objetivo esencial..." (pág. 3). Dicho de otro modo, es orientar la educación hacia el pleno desarrollo del individuo, incluyendo derechos humanos con las respectivas libertades fundamentales, basadas en las finalidades educativas.

Ahora bien, la responsabilidad social en la universidad en tiempos de posmodernidad genera nociones, generalidades que pueden ocasionar críticas o diatribas, si enfocamos la universidad desde segmentos dirigidos a la consecución de propósitos en mejora de los contextos sociales que incumben a su organización educativa. Asimismo, a la universidad, se le confiere el título de crear ideas, sustentadas en el cumplimiento de roles actuales planteados como es de esperar. Al respecto, Jiménez (2017), expone:

La universidad en la búsqueda del desarrollo y divulgación del 
conocimiento en los campos del saber, debe cumplir con las funciones de docencia, extensión, investigación y gestión, desde las cuales pueda asumir el proceso académico que coadyuve al mejoramiento del potencial humano... (pág. 379).

En ese orden de ideas, la universidad para dar cumplimiento con saberes debe dar fiel desempeño a las funciones que les competen, dando énfasis en este caso, a la ocupación de la extensión y así observar generalidades con la vinculación universitaria asumida como proceso imaginado en la orientación hacia la construcción de aprendizajes desde el enfoque constructivista, lo que permite, en este momento una manera diferente de pensamiento y mediación de epistemes ante la complejidad de los contextos, inherentes a la educación universitaria en el caso mostrado.

Visto así desde el ámbito educativo, el estudiante en este momento que se integra a la función social de la universidad con los contextos comunitarios, posee un sustento legal en la Ley del Servicio Comunitario del Estudiante de Educación Universitaria, (LSCES, 2005). Se enfatiza en las comunidades académicas, que no poseen sentido de pertenencia y están deshumanizadas en la realidad universidad-comunidad, lo que dificulta el desempeño social, el cual consiste en difundir el conocimiento que en ellas se genera, para contribuir con el desarrollo social de su entorno. Aunado a esto, la universidad es más un ideal, un deber ser, que una organización específica de la que es ineludible dar cuenta analíticamente. Por esto, Kuhn (1971), citado por Arana (2012), precisa que:

Las comunidades científicas y académicas comparten una matriz disciplinaria, la cual puede ser entendida como el conjunto de elementos en torno a los cuales se constituye la unidad de las mismas. Una particularidad importante de estas comunidades es que son precisamente ellas quienes producen y validan por consenso el conocimiento científico / académico (pág. 25). 
De igual manera, se visualiza poca participación de parte de la comunidad social en las actividades que realiza la universidad, las cuales, aunque pudieran estar dirigidas en su beneficio, no les produce ningún interés ya que, no se consideran parte importante del accionar universitario, es decir, no existe vinculación entre ambas.

En esta descripción del problema, también se percibe, que las políticas de Estado desmotivan el interés de los docentes por el desempeño investigativo, así como la misma universidad no los incentivan a una mayor dedicación por esta actividad, sin otorgarle el reconocimiento tanto económico como social y profesional suficiente para que dediquen todo su interés en el logro de la difusión del conocimiento hacia el beneficio de la comunidad a través de una verdadera integración.

La situación descrita, en cuanto al beneficio de esta vinculación en los espacios sociales admite con urgencia una reforma para la realidad actual, donde se puedan llevar a cabo procesos como edificar, planear, constituir, componer y valorar en acción conjunta con las comunidades donde las acciones sean desplegadas. Observando estas realidades, a partir de la reflexión de actores sociales, un número significativo tiene problemas al momento de reconocer requerimientos los cuales esbozan todo tipo de reforma.

A favor de vincular los entes universitarios y comunitarios aunados a esto sus contradicciones obtienen representación institucional para ser llevadas a la práctica. Como alternativas de solución ante esta problemática planteada, se pueden mencionar lo relativo a la gestión interna de la universidad, debe estar enfocada a su transformación en pequeña comunidad con pertinencia social, es decir socialmente ejemplar, con doble instrucción: el discípulo adquiere en el entorno universitario las herramientas para el desempeño de su carrera asimilando de ésta su dimensión axiológica, respecto a los valores morales. 
Respecto, a lo educacional la misión universitaria, debe estar dirigida a facultar a los docentes en cuanto a la Responsabilidad Social Universitaria y promover en las especialidades el aprendizaje basado en proyectos de carácter social, abriendo el salón de clase hacia la comunidad social como fuente de enseñanza significativa y práctica dedicada a la solución de problemas reales

Desde estas ideas, pienso que es el momento de desarrollar todo un planteamiento $\mathrm{y} / \mathrm{o}$ proceso de investigación que sirva de respaldo a la consideración de la responsabilidad social como una variable educativa, que juega un papel activo en el desarrollo del desempeño del docente universitario que sirva para concretar nuestro objeto de investigación. Por ello, la inquietud consiste en investigar los roles a cumplir en su desempeño la universidad referente a la vinculación con la comunidad.

\section{Conclusiones}

De manera conclusiva, en nuestra opinión, todo esto es un devenir de una reflexión a conciencia de las instituciones universitarias, respecto a su actuación en los ámbitos sociales, para sintetizar responsablemente el incumplimiento en dificultades habituales dirigidas a la socialización del individuo.

Todo se circunscribe, en un recuadro de principios éticos, que dan identidad al ejercicio de la función docente. En consecuencia, se dejó claro que el profesor que se precie de tener ética y moral, debe actuar con responsabilidad en este caso, dirigida al entorno social que circunda los espacios universitarios donde desempeña su loable labor. Asumiendo, que su deber ser es compartir con sus estudiantes su ser, como su hacer y el conocimiento que ostenta.

Por esto, el compromiso de la extensión universitaria beneficia la responsabilidad ética-social del estudiante en y con la comunidad inmersa en 
el entorno. Además, esta extensión cumple función concienciadora y crítica, al situar la problemática comunitaria frente a las universidades, generando reflexiones y generalidades en el rol que cumple a favor de la transformación social.

\section{Referencias}

Arana, M. (2012). Comunidades Académicas Universitarias y Políticas Públicas en Educación Superior en Argentina. Una perspectiva Antropológica (1992-2012). Tesis Doctoral. España: Universidad Autónoma de Madrid, págs. 332. Recuperado de:

https://repositorio.uam.es/bitstream/handle/10486/660330/arana llera marta beatriz.pdf?sequenc

Castro, S., \& Guzmán, B. (2005) Los Estilos de Aprendizaje en la Enseñanza y el Aprendizaje: Una propuesta para su Implementación. Revista de Investigación, (58), 83-102, ISSN: 07980329. Caracas, Venezuela: Universidad Pedagógica Experimental Libertador. Recuperado de:

http://www.redalyc.org/pdf/3761/376140372005.pdf

Cruz, C. (2010). La Responsabilidad de la universidad en la sociedad que la acoge. ¿Complementariedad o antagonismo? Responsabilidad social universitaria, ISBN: 978-84-9745-423-0, págs. 25-45. España: Editores Netbiblo.

Gaete, R. (2012). Responsabilidad Social Universitaria: Una nueva mirada a la relación de la universidad con la sociedad desde la perspectiva de las partes interesadas. Un estudio de caso. Tesis Doctoral. España: Universidad de Valladolid. Recuperado de:

http://uvadoc.uva.es/handle/10324/923

Jiménez, M. (2017). Hermeneusis Ontológica del Estudiante Universitario. Revista Scientific, 2(5), 377-395. Recuperado de: 
https://doi.org/10.29394/scientific.issn.2542-2987.2017.2.5.20.377-395 LSCES (2005). Ley de Servicio Comunitario del Estudiante de Educación

Superior. Gaceta Oficial N. 38.272 del 14 de septiembre. Carcas, Venezuela: Asamblea Nacional de la República Bolivariana de Venezuela. Recuperado de:

http://www.ciens.ucv.ve/ciens/servicio comunitario/normativa/LeydeSe rvicioComunitarioGaceta.pdf

ONU (1933). Conferencia Mundial sobre Derechos Humanos. Informe A/CONF.157/23. Viena: Organización de las Naciones Unidas, ONUECLAC.

Rodríguez, J. (2010). Responsabilidad social universitaria. Del discurso simbólico a los desafíos reales. Responsabilidad social universitaria, ISBN: 978-84-9745-423-0, págs. 3-24. España: Editores Netbiblo.

Secchi, D. (2007). Utilitarian, managerial and relational theories of corporate social responsibility. IJMR International Journal of Management Reviews, 9(4):347-373, ISSN: 1468-2370. Recuperado de: https://doi.org/10.1111/j.1468-2370.2007.00215.x

UNESCO (2008). Estándares de Competencias en TIC para Docentes. Londres: Organización de las Naciones Unidas para la Educación, la Ciencia y la Cultura. Recuperado de:

http://eduteka.icesi.edu.co/pdfdir/UNESCOEstandaresDocentes.pdf 


\section{Mariella Fidelina Rojas Zerpa \\ e-mail: mariella1117@gmail.com}

Nacida en Caracas, Venezuela. Poseo Título de

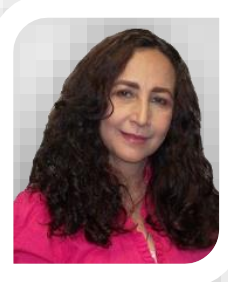

Especialista en Evaluación Educacional, 1er Lugar de la Promoción. Integrante del Cuadro de Honor, de la Universidad Valle del Momboy, Estado Trujillo. Profesora de Historia y Ciencias Sociales en Escuela Nacional Bolivariana "María Pereira" de Daza en Barquisimeto, Lara. Profesora Invitada de la Universidad Valle del Momboy desde el 2013, hasta la actualidad, en las asignaturas: Bases Teóricas de la Didáctica, Impacto del Desempeño Laboral Docente y Marco Teórico de la Planificación. 